\title{
Concepts for the Temporal Characterization of Short Optical Pulses
}

\author{
Christophe Dorrer \\ Bell Laboratories, Lucent Technologies, 791 Keyport-Holmdel Road, Holmdel, NJ 07733, USA \\ Email:dorrer@lucent.com
}

\section{Ian A. Walmsley}

Clarendon Laboratory, Parks Road, Oxford OX1 3PU, United Kingdom

Email:walmsley@physics.ox.ac.uk

Received 1 April 2004; Revised 15 September 2004

\begin{abstract}
Methods for the characterization of the time-dependent electric field of short optical pulses are reviewed. The representation of these pulses in terms of correlation functions and time-frequency distributions is discussed, and the strategies for their characterization are explained using these representations. Examples of the experimental implementations of the concepts of spectrography, interferometry, and tomography for the characterization of pulses in the optical telecommunications environment are presented.
\end{abstract}

Keywords and phrases: optical pulse characterization, time-frequency distribution, Wigner function, spectrogram, tomography, interferometry.

\section{INTRODUCTION}

Ultrashort optical pulses are used in areas of science and engineering as diverse as spectroscopy, medical research, plasma physics, quantum optics, and optical telecommunications. In optical telecommunications, information is encoded in the amplitude and/or phase of an optical wave [1]. While information encoding in digital telecommunications is based on a finite number of values of a physical quantity (e.g., the presence or absence of energy in a given bit slot), the ability to measure in detail the waveform of the optical wave itself is crucial for optimizing the properties of the systems that generate the signal, and understanding the linear and nonlinear properties of the systems through which the pulses propagate. This information is critical in developing strategies to overcome the current limitations of current optical networks. For example, dispersion management compensates for the chromatic dispersion induced by linear propagation and can also be used to mitigate nonlinear effects. Similarly, the phase distortion imposed on a pulse by the modulators used in carving the pulse out of a cw- or quasi-cw source can impact the propagation of the pulse. Finally, measurements of the electric field can be used to characterize the linear or nonlinear properties of a device. There are various approaches for temporal waveform measurements. We only consider here techniques that provide self-referencing characterization of an unknown pulse or a train of unknown but identical pulses, that is, that do not use a well-characterized pulse as a reference. While test-plus-reference techniques, such as spectral interferometry $[2,3,4]$, can be easier to implement in some cases, they require a well-characterized reference pulse mutually coherent with the pulse under test. Although this can be difficult to achieve over long distances, they have been used to characterize pulses in the telecommunication environment [5]. We will not deal either with sampling techniques. These techniques can provide samples of the temporal intensity of a source by fast photodetection and electronics or by nonlinear interaction with a short sampling pulse (nonlinear optical sampling) [6]. The technique of linear optical sampling [7] is also sensitive to the electric field of the source (i.e., it can measure samples of the intensity and phase of the source under test), and can therefore be used to measure constellation diagrams [8]. These techniques are particularly useful when dealing with a data-encoded optical source because of the randomness of the data stream, but they constitute a class of their own that is beyond the scope of this paper. The concepts presented here apply generally to the characterization of the temporal electric field of short optical pulses, although the details of experimental implementations are strongly dependent upon the domain of application.

Since ultrafast optical pulses are faster than the fastest electronic detection devices, they present a considerable challenge for measurement. An important additional limitation is that photodetectors respond to the intensity of the pulse, 
so their output contains no information about the phase of the incident radiation. To overcome these limitations, a combination of ancillary filters can be used. The data are simply the photocurrent recorded by a time-integrating detector as a function of the parameters of the filters. These might be, for example, the passband frequency for a spectrometer (a time-stationary linear filter), the modulation index for a phase modulator (a time-nonstationary linear filter), or the relative delay between the pulse under test and the modulation induced by an electroabsorption modulator (also a time-nonstationary linear filter).

There are a number of quite general strategies for characterizing the electric field of an optical pulse using such filters. These belong to one of three categories: spectrographic, tomographic, or interferometric. The categories are distinguished by the procedure required for reconstructing the amplitude and the phase of the field from the recorded data $[9,10]$. Since the analytic signal of the field is a complex function of one real variable, time, with finite support, the data must contain a finite set of complex numbers sampling the field at a finite set of time points, or equivalently a finite set of frequency points. It can sometimes be fruitful, however, to reconstruct pulses by sampling a time-frequency representation of the pulse (or its equivalent correlation function). In this case a two-dimensional set of data is obtained, from which an inversion algorithm reconstructs an estimate of the field. This is typically the case for spectrography, which makes use of a time-frequency distribution, and requires sophisticated iterative data inversion algorithms to reconstruct the field. Tomography also requires a large data set, in the form of a large number of modulated pulse spectra, but the inversion is direct (noniterative). Interferometry, in contrast, measures only a one-dimensional data set and uses direct data inversion to reconstruct the field.

In this paper, we provide examples of each of these methods that are relevant to optical telecommunications. In Section 2, we first discuss the representation of the electric field of a pulse or train of pulses, and how the various measurement techniques sample the field. Then, in Section 3, we illustrate both the data acquisition and inversion for each method.

\section{REPRESENTING LIGHT PULSES}

The fundamental quantity describing an isolated, individual pulse of light is the real electric field. This is a function of time and space, or equivalently frequency and wavevector. The spatial dependence of the field is often assumed uniform. This assumption is valid for optical fiber-based telecommunications if the field occupies the lowest-order mode of the fiber. Therefore in this paper, we concentrate on determining the time dependence of the electric field.

In practice, it is often difficult to characterize a single pulse, and one deals with a train of pulses instead of a single pulse. One must be careful in specifying a field for such an ensemble. If all of the pulses in the train are identical, the ensemble is deemed coherent, and the underlying electric field of an individual remains the quantity of interest. If, on the other hand, the electric field is stochastic, fluctuating from pulse to pulse, the ensemble is said to be partially coherent. When this is the case, the amplitude and phase of the electric field of an individual pulse brings little information on the train of pulses, and pulse characterization involves measurements of the statistical properties of the ensemble, for example via the two-time or two-frequency correlation function. We note that a data-modulated train of pulses is not coherent if the data modulation is random (which is the case for a deployed communication system).

\subsection{Describing an optical pulse by its analytic signal}

The real electric field, $\varepsilon(t)$, underlying an optical pulse is twice the real part of its analytic signal $E(t): \varepsilon(t)=2 \times$ $\operatorname{Re}[E(t)]$. The analytic signal is the single-sided inverse Fourier transform of the Fourier transform of the field,

$$
E(t)=\frac{1}{\sqrt{2 \pi}} \int_{0}^{\infty} d \omega \widetilde{\varepsilon}(\omega) \exp [-i \omega t]
$$

where

$$
\widetilde{\mathcal{\varepsilon}}(\omega)=\frac{1}{\sqrt{2 \pi}} \int_{-\infty}^{\infty} d t \varepsilon(t) \exp [i \omega t] .
$$

The electric field is considered to have compact support in the time domain, and is further assumed to have no spectral component at $\omega=0$ so $\widetilde{\varepsilon}(0)=0$ (since a pulse propagating in a charge-free region of space has no dc spectral component, the electric field must have zero area). The analytic signal is complex and therefore can be expressed uniquely in terms of an amplitude and phase:

$$
E(t)=|E(t)| \exp \left[i \phi_{t}(t)\right] \exp \left[i \phi_{0}\right] \exp \left[-i \omega_{0} t\right],
$$

where $|E(t)|$ is the time-dependent envelope, $\omega_{0}$ is the carrier frequency (usually chosen near the center of the pulse spectrum), $\phi_{t}(t)$ is the time-dependent phase, and $\phi_{0}$ a constant. The square of the envelope, $|E(t)|^{2}$, is the time-dependent intensity of the pulse which could be measured by a detector of sufficient bandwidth. The time-dependent phase accounts for the occurrence of different frequencies at different times, and the instantaneous frequency is usually defined as $-\partial \phi_{t} / \partial t$. As an example, Figure 1 shows the temporal intensity and phase of a pair of chirped Gaussian pulses. The timedependent phase structure is indicative of a variation of the instantaneous frequency across the pulse.

The frequency representation of the analytic signal is the Fourier transform of $E(t)$,

$$
\begin{aligned}
\widetilde{E}(\omega) & =|\widetilde{E}(\omega)| \cdot \exp \left[i \phi_{\omega}(\omega)\right] \\
& =\frac{1}{\sqrt{2 \pi}} \int_{-\infty}^{\infty} d t E(t) \exp [i \omega t] \\
& = \begin{cases}\widetilde{\varepsilon}(\omega), & \omega>0, \\
0, & \omega \leq 0 .\end{cases}
\end{aligned}
$$




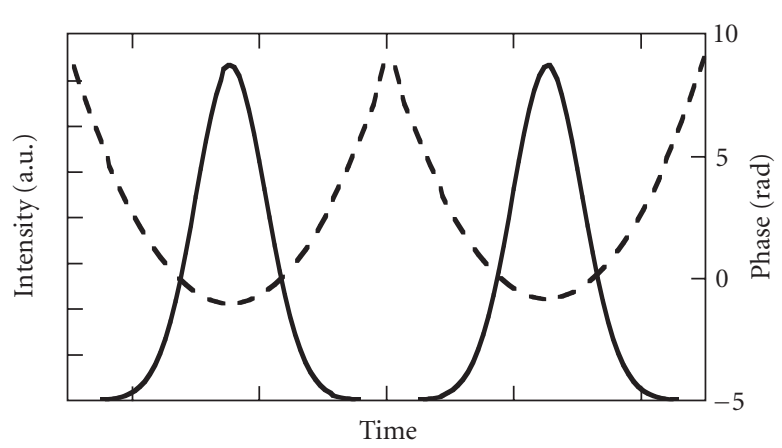

(a)

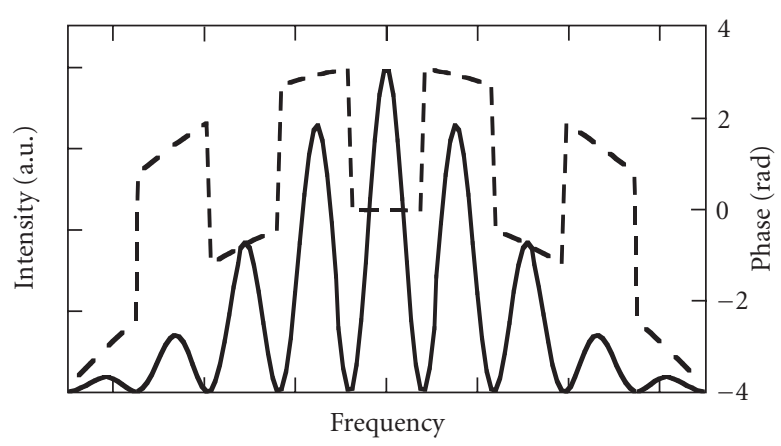

(b)

FIGURE 1: Intensity and phase (resp., continuous and dashed line) of a pair of chirped Gaussian pulses in (a) the temporal domain and (b) the spectral domain.

Here $|\widetilde{E}(\omega)|$ is the spectral amplitude and $\phi_{\omega}(\omega)$ is the spectral phase. The square of the spectral amplitude, $|\widetilde{E}(\omega)|^{2}$, is the spectral intensity. Strictly speaking this quantity is the spectral flux-the quantity measured in the familiar way by means of a spectrometer followed by a photodetector. The spectral phase describes the relative phases between each of the frequencies, and the group delay for frequency $\omega$ is usually defined as $\partial \phi_{\omega} / \partial \omega$.

The sampling requirement for the reconstruction of the electric field is given by the Whittaker-Shannon theorem [11], which asserts that if the field has compact support in the time domain over a range $\Delta t$, then a sampling of $\widetilde{E}(\omega)$ at the Nyquist frequency rate of $2 \pi / \Delta t$ is sufficient for reconstructing the analytic signal $E(t)$ and consequently the electric field $\varepsilon(t)$ exactly.

Figure 1 shows the spectral intensity and spectral phase of the chirped pair of Gaussian pulses. The spectral fringes have a period of the inverse of the temporal separation of the pair of pulses. While the spectrum can reveal some properties of the waveform, both the spectral intensity and phase must be measured to fully characterize the electric field.

\subsection{The two-time correlation function and its phase-space representations}

If a measurement relies on averaging the detected signal over a train of pulses, then it is necessary to define the properties of the pulses in a different way. Although it is formally quite difficult to formulate rigorously even the simplest of concepts, such as the spectrum [12], for a nonstationary field, a simple-minded approach can be fruitful. If each pulse in the train is an independent realization of a stochastic ensemble, then the time average is equivalent to an ensemble average by definition. This enables the coherence of the train to be defined operationally in a reasonable way. It is important, though, to realize that the electric field amplitude and phase of an individual pulse does not bring significant information about the train of pulses and pulse characterization efforts must ultimately be directed toward measurement of the ensemble statistics.
The simplest quantity that quantifies the statistical properties of the ensemble is the nonstationary two-time field correlation function

$$
C\left(t_{1}, t_{2}\right)=\left\langle E\left(t_{1}\right) E^{*}\left(t_{2}\right)\right\rangle,
$$

where the angle brackets indicate an average over the ensemble of pulses, each of the electric fields being defined with respect to a local time frame. With this definition of the ensemble, we do not need to adopt procedures along the lines of those developed by Wiener and Khintchine [13] to define the correlation function.

$C\left(t_{1}, t_{2}\right)$ provides a quantitative description of fluctuations from pulse to pulse in the electric field at times $t_{1}$ relative to those at times $t_{2}$. This is a complete description of the pulse ensemble so long as the fluctuations obey normal (or Gaussian) statistics. If not, then it is the simplest of a hierarchy of multitime correlation functions defining the ensemble. Furthermore, for a train of identical pulses, $C\left(t_{1}, t_{2}\right)$ factorizes into $E\left(t_{1}\right) E^{*}\left(t_{2}\right)$ and the electric field amplitude and phase are readily obtained.

It is frequently useful to work with a variation of the correlation function that uses a two-dimensional space of time and frequency-the chronocyclic phase-space. The intuitive concept of chirp (i.e., time-dependent frequency in the pulse) can be most easily seen within this space. The relationships between the two-time correlation function and the chronocyclic (time/frequency) and frequency-domain representations of the ensemble may be derived by rewriting (5) in terms of a center-time coordinate, $t$, and a difference-time coordinate, $\Delta t$ :

$$
C(t, \Delta t)=C\left(t_{1}, t_{2}\right),
$$

where $t=\left(t_{1}+t_{2}\right) / 2$ and $\Delta t=t_{1}-t_{2}$. The two-frequency field correlation function is obtained by taking the twodimensional Fourier transform of the two-time correlation function

$$
\widetilde{\widetilde{C}}(\Delta \omega, \omega)=\frac{1}{2 \pi} \iint d t d \Delta t C(t, \Delta t) \exp [i(t \Delta \omega+\Delta t \omega)] .
$$




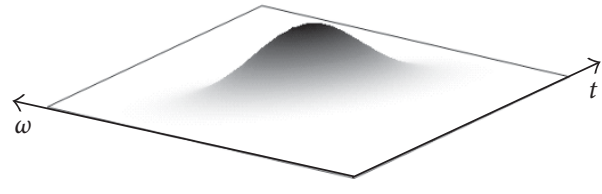

(a)

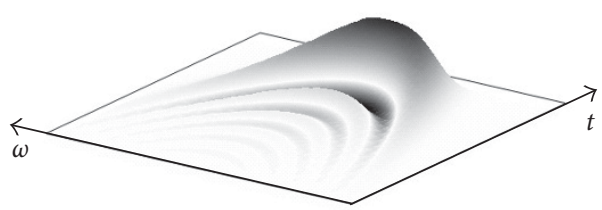

(b)

FIGURE 2: Wigner function of a pulse with (a) linear chirp and (b) quadratic chirp.

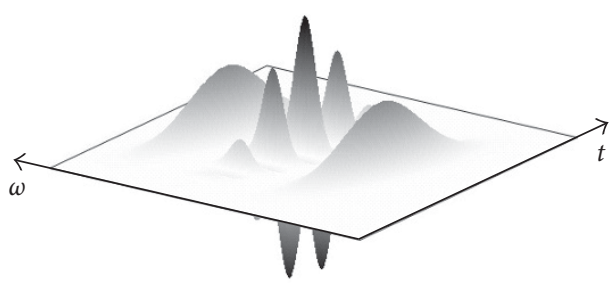

(a)

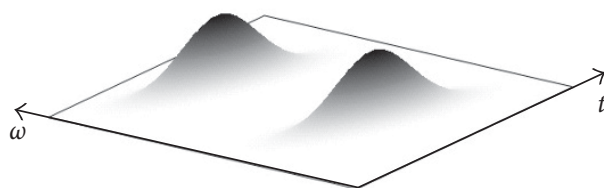

(b)

FIGURE 3: Wigner function of the pair of chirped Gaussian pulses when the two pulses are (a) mutually coherent and (b) mutually incoherent.

The center-frequency and difference-frequency coordinates in (7) are given by $\omega=\left(\omega_{1}+\omega_{2}\right) / 2$ and $\Delta \omega=\omega_{1}-\omega_{2}$, respectively. The pulse ensemble may also be represented within the chronocyclic phase spaces defined by the complimentary variables $t, \omega$ and $\Delta \omega, \Delta t$. The chronocyclic Wigner function, $W(t, \omega)$, and ambiguity or Wigner characteristic function, $A(\Delta \omega, \Delta t)$, provide two particularly useful descriptions of the pulse train statistics in these spaces. The relationship between the various representations of the correlation function has been discussed in the context of spatially localized fields in [14], and in the context of signal analysis in [15]. Chronocyclic phase-space distributions have found increasing application in ultrafast optics. The Wigner function has been described in $[16,17,18]$, and the Page distribution in [19]. Another distribution of interest is the ambiguity function that is used in radar technology [20]. These functions are also used in many areas of physics and engineering, and their relations and properties are discussed in [21]. The Wigner function is obtained by taking the one-dimensional Fourier transform of $C(t, \Delta t)$ over the time-difference coordinate

$$
W(t, \omega)=\frac{1}{\sqrt{2 \pi}} \int d \Delta t C(t, \Delta t) \exp [i \omega \Delta t]
$$

whereas the ambiguity function is obtained from $C(t, \Delta t)$ by performing the Fourier transform over the average-time coordinate

$$
A(\Delta \omega, \Delta t)=\frac{1}{\sqrt{2 \pi}} \int d t C(t, \Delta t) \exp [i \Delta \omega t]
$$

These representations are uniquely related to one another by Fourier transformations. The Wigner function has many interesting properties, for example, the ability to represent a chirp, as can be seen in Figure 2a. It is a real, but not necessarily positive function, which complicates its interpretation as a density function in the time-frequency space. For example, the Wigner function of a Gaussian pulse with a quadratic chirp (i.e., a third-order spectral phase), as displayed in Figure 2b, is negative over a significant portion of the chronocyclic space. An example of the Wigner function of the pair of chirped Gaussian pulses in Figure 1 is shown in Figure 3a. The side lobes are the phase-space representations of the individual pulses, and the cross-terms between the two fields lead to a central feature with fringes that indicate that the pulses have a definite phase relation to one another-they are coherent. The Wigner function for an incoherent pair of pulses is also show in Figure 3b. In this case the ensemble has a random phase between the pair, which causes a washing out of the interference pattern when the signal is averaged over the ensemble. The coherence can be quantified using the Wigner function, as explained in the next section.

Information regarding the shapes of ultrafast optical pulses is generally inferred from the output of a square-law detector after some filtering. Therefore it is of practical interest to establish the relationship between measurable detector output and the various descriptions of the pulse ensemble. The average pulse time-dependent intensity is obtained from the two-time correlation function by setting $\Delta t=0$. Alternatively, it is a projection of the Wigner function onto the frequency axis, or the Fourier transform of the $\Delta t=0$ line of the ambiguity function

$$
\begin{aligned}
I(t) & =C(t, 0) \\
& =\int d \omega W(t, \omega) \\
& =\int d \Delta \omega A(\Delta \omega, 0) \exp [-i \Delta \omega t] .
\end{aligned}
$$


Furthermore, the average pulse spectral intensity is obtained from the two-frequency correlation function by setting $\Delta \omega=$ 0 , or by projecting the Wigner function onto the frequency axis, or by taking the Fourier transform of the $\Delta \omega=0$ line of the ambiguity function:

$$
\begin{aligned}
I(\omega) & =\widetilde{\widetilde{C}}(0, \omega) \\
& =\int d t W(t, \omega) \\
& =\int d \Delta t A(0, \Delta t) \exp [-i \Delta t \omega]
\end{aligned}
$$

It is important to recognize that the various time-frequency distributions and their relations are central to the characterization of pulses in the optical domain, since they are simply related to the measured data. In optics, direct measurement of the waveform is not possible. This is in contrast to the more usual application of these distributions in signal processing, where they are commonly used as mathematical tools for signal representation, for example, to track the presence of various instantaneous frequencies in a known (measured) sound waveform.

\subsection{The integral degree of coherence: when is a pulse field useful?}

Since a field amplitude and phase may be defined in a unique way only for identical pulses, it is important to quantify the degree to which the pulses in the ensemble are alike. A useful quantity for this purpose is the integral degree of coherence, $\mu$. $\mu$ is readily derived from the time-domain analogue of Born and Wolf's [13] degree of coherence $\gamma(t+\Delta t / 2, t-\Delta t / 2)$, defined as

$$
\gamma\left(t+\frac{\Delta t}{2}, t-\frac{\Delta t}{2}\right)=\frac{C(t, \Delta t)}{[C(t+\Delta t / 2,0) C(t-\Delta t / 2,0)]^{1 / 2}} .
$$

Using the Schwarz inequality, it is straightforward to show that $0 \leq|\gamma(t+\Delta t / 2, t-\Delta t / 2)| \leq 1$. Consider the $n$ dimensional vectors $\vec{a}$ and $\vec{b}$, with components $\left\{a_{i}\right\}$ and $\left\{b_{i}\right\}$ where $i \in(1, n)$. Then

$$
0 \leq\left|\sum_{i=1}^{n} a_{i}^{*} b_{i}\right|^{2} \leq\left(\sum_{i=1}^{n} a_{i}^{*} a_{i}\right)\left(\sum_{i=1}^{n} b_{i}^{*} b_{i}\right) .
$$

Allowing $a_{i}=E_{i}(t-\Delta t / 2) / \sqrt{n}$ and $b_{i}=E_{i}(t+\Delta t / 2) / \sqrt{n}$, that is, the $i$ th realization of the field amplitude at times $t-\Delta t / 2$ and $t+\Delta t / 2$, respectively, it is clear that

$$
\begin{aligned}
0 \leq & \left|\frac{1}{n} \sum_{i=1}^{n} E_{i}^{*}\left(t-\frac{\Delta t}{2}\right) E_{i}\left(t+\frac{\Delta t}{2}\right)\right|^{2} \\
\leq & \left(\frac{1}{n} \sum_{i=1}^{n} E_{i}^{*}\left(t+\frac{\Delta t}{2}\right) E_{i}\left(t+\frac{\Delta t}{2}\right)\right) \\
& \times\left(\frac{1}{n} \sum_{i=1}^{n} E_{i}^{*}\left(t-\frac{\Delta t}{2}\right) E_{i}\left(t-\frac{\Delta t}{2}\right)\right) .
\end{aligned}
$$

In the limit that $n \rightarrow \infty$, the summations in (14) lead to an average over the ensemble and the inequality simplifies to

$$
0 \leq|C(t, \Delta t)|^{2} \leq C\left(t+\frac{\Delta t}{2}, 0\right) C\left(t-\frac{\Delta t}{2}, 0\right) .
$$

The upper and lower bounds on the degree of coherence follow from (15). However, it is difficult to determine $\gamma(t+$ $\Delta t / 2, t-\Delta t / 2)$ experimentally since it becomes singular for times at which $C(t, \Delta t)$ is zero. A practically more useful definition is offered by integrating equation (15) over the entire $t, \Delta t$ space, and dividing by the quantity on the right-hand side, leading to the integral degree of coherence, $\mu$,

$$
0 \leq \mu=\frac{\iint d t d \Delta t|C(t, \Delta t)|^{2}}{\left[\int d t C(t, 0)\right]^{2}} \leq 1
$$

Equivalent relations follow for the frequency domain and chronocyclic representations, for example, in the case of the ambiguity function

$$
\mu=\frac{\iint d \Delta t d \Delta \omega|A(\Delta \omega, \Delta t)|^{2}}{[A(0,0)]^{2}} .
$$

An integral degree of coherence less than one corresponds to a partially coherent train in which the pulse shape and/or phase fluctuate, in which case $C(t, \Delta t)$ is the fundamental quantity of interest. When $\mu=1$ the ensemble is said to be fully coherent (the pulses in the ensemble are identical within a constant phase factor) and $C(t, \Delta t)$ factorizes. In the latter case the electric field becomes the fundamental quantity of interest and is readily retrieved from the two-time correlation function using

$$
|E(t)|=\sqrt{C(t, 0)}
$$

and with $t_{2}$ held fixed,

$$
\operatorname{Arg}[E(t)]=\tan ^{-1}\left[\frac{\operatorname{Im} C\left(\left(t+t_{2}\right) / 2, t-t_{2}\right)}{\operatorname{Re} C\left(\left(t+t_{2}\right) / 2, t-t_{2}\right)}\right]+\phi_{0}
$$

where $\phi_{0}$ is an undetermined constant. For example, the integral degree of coherence corresponding to the Wigner function in Figure $3 \mathrm{a}$ is equal to one, while that of the Wigner function in Figure $3 \mathrm{~b}$ is equal to 0.5 . It is important to note that (18) and (19) are valid only if the integral degree of coherence has been explicitly demonstrated to be equal to unity, which of course requires that the two-time correlation function or equivalent representation in frequency or phase space be measured. Thus in cases where an ensemble or train of pulses, rather than an individual pulse, is used for application or experimentation, pulse-shape characterization efforts must ultimately be directed toward measurement of the ensemble statistics. 
For a coherent train of pulses, (18) and (19) reveal that the electric field is retrieved from a single line of the correlation function. Hence, if the ensemble is assumed a priori to be coherent, the amount of collected data can be greatly reduced. This is a luxury afforded only to measurement techniques that directly measure one of the correlation functions.

\subsection{Measurement strategies}

The electric field of optical pulses can be characterized using various strategies, derived from, and with implications for, other measurement problems, such as wavefront diagnosis and quantum state reconstruction. These strategies can be organized into phase-space techniques, that is, techniques that attempt to measure either the Wigner or ambiguity function by exploring the entire two-dimensional chronocyclic phase space, and direct techniques, that obtain the electric field of a coherent field from a single slice of a secondorder correlation function.

The minimal requirement for the complete exploration of the chronocyclic space required in phase-space techniques is the presence of two filters. The analysis details of phasespace techniques are found in [9]. There are two subclasses of phase-space techniques; those that make simultaneous measurements of the complementary variables in an attempt to reconstruct one of the phase-space distributions, and those that record marginals of the Wigner function after rotation in the phase space, from which the Wigner function can be obtained. The former method is known as spectrographic while the latter is referred to as tomographic. Spectrography is discussed in Sections 3.1 and 3.2, and tomography is discussed in Sections 3.5 and 3.6.

In contrast, direct techniques do not require this complete exploration of the phase space occupied by the correlation function. This is a significant advantage of direct techniques compared to phase-space techniques. Moreover, if the pulse train is assumed a priori to consist of identical pulses, as is most always assumed in reconstructing pulses from spectrographic or tomographic data, only one slice of the correlation function is required to obtain the amplitude and phase of the electric field [10]. Such slices are precisely what is measured in interferometry. This is usually achieved by mixing the field under test with a modified version of itself, or more generally by mixing two modified versions of the field under test. Thus, while phase-space techniques must explore the entire chronocyclic space even when the electric field is the fundamental quantity of interest, direct techniques need only return a single slice of the correlation function in order to construct the simpler quantity. Roughly speaking, if one wishes to reconstruct the field at $N$ time points, then at least $2 \mathrm{~N}$ independent data points are required. While direct techniques are capable of reconstructing the field by recording only the necessary $2 \mathrm{~N}$ points, phase-space techniques require the measurement of $N^{2}$ points. Of course, an overcomplete data set is available from direct measurement of the entire correlation function as well. Direct interferometric techniques are discussed in Sections 3.3 and 3.4.

\section{SELF-REFERENCING TEMPORAL CHARACTERIZA- TION OF SHORT OPTICAL PULSES}

From a practical point of view, one first measures an experimental trace (e.g., the current from a photodiode as a function of various parameters of the experimental setup, e.g., the central frequency of a passband spectral filter), then applies a set of mathematical operations to the measured data in order to reconstruct the electric field. The design of the experimental setup and the type of experimental trace determine the recovery algorithm, and more generally the possibility of such recovery. In this section we take a closer look at the concepts of spectrography, tomography, and interferometry, and examples of experimental implementations and results are given.

\subsection{Spectrography}

Spectrographic techniques make use of two sequential filters, one time-stationary (spectral filter) and one timenonstationary (time gate) followed by a square-law detector (Figure 4). The recorded signal is either a measure of the spectrum of a series of time slices (spectrogram) or a measure of the time of arrival of a series of spectral slices (sonogram) depending upon the ordering of the filters. There is no difference in principle between the two possible filter orderings and thus this type of apparatus should be thought of as one that makes simultaneous measurements of the conjugate variables rather than sequential measurements. Since precise measurements of the conjugate variables cannot be made simultaneously, a spectrographic apparatus can measure only a smoothed out version of the Wigner function - the Wigner function convolved with an apparatus blurring or window function. In principle, if the window function is known, the Wigner function itself can be obtained via deconvolution but this is usually impractical because of the severe signal-tonoise requirements. Thus the spectrographic class of phasespace pulse characterization techniques supply only qualitative insight into pulse train statistics. However, if the pulses in the ensemble are assumed a priori to be identical, the resultant two-dimensional phase-retrieval problem can be solved iteratively. The success of this approach has been extensively demonstrated in the technique of frequency-resolved optical gating (FROG) [22].

A typical implementation of spectrography uses a temporal gate for the signal under test (e.g., the action of the pulse under test with one or several other pulses in a nonlinear optical medium [22], or a "shutter" function provided by a temporal modulator [23]) and a device capable of measuring the optical spectrum (e.g., an optical spectrum analyzer based on a diffraction grating and imaging optics, or a scanning Fabry-Perot etalon, together with a time-integrating photodiode). The spectrogram of the electric field of the test pulse is obtained by measuring the optical spectrum of the pulse after temporal gating for various relative delays between the pulse and the gate. The experimental trace is therefore

$$
S(\omega, \tau)=\left|\int E(t) R(t-\tau) \exp (i \omega t) d t\right|^{2},
$$




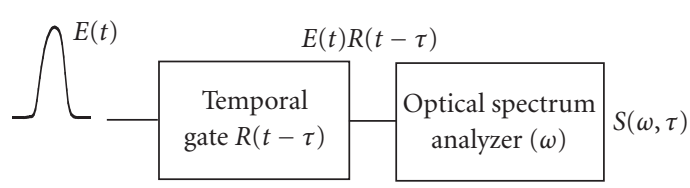

(a)

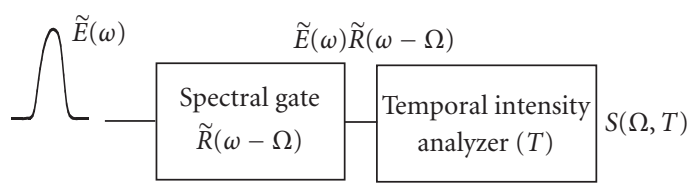

(b)

FIgURE 4: Conceptual implementation of (a) a spectrogram and (b) sonogram.

where $\omega$ is the optical frequency and $\tau$ the relative delay between the gate and the test pulse. It is important that the resolution of the spectral filter is very high in order to ensure that the measured trace is effectively the spectrogram of the test pulse.

A sonogram can be measured by reversing the order of the temporal and spectral gate $[24,25]$. Typically, the pulse is first spectrally filtered using a spectrometer with variable central frequency $\Omega$. The temporal intensity of the filtered pulse is then measured, and the sonogram is constructed as the set of the measured temporal intensities for various central frequencies $\Omega$ :

$$
S(\Omega, T)=\left|\int \widetilde{E}(\omega) \widetilde{R}(\omega-\Omega) \exp (-i \omega T) d \omega\right|^{2} .
$$

In this case the temporal resolution should be very high to ensure that the measured trace is a true sonogram. In practice, one usually implements the sonogram by means of a nonlinear cross-correlation of the spectrally gated signal with the test pulse, which has a shorter duration than the filtered pulse. Therefore, the experimental trace is given by a convolution of the sonogram of (21) with the unknown temporal intensity of the pulse under test, a fact that can be included in the inversion algorithm [25].

It can be shown that the spectrogram is the correlation of the Wigner function of the pulse with the Wigner function of the gate with a change of sign on the frequency variable [15]:

$$
S(\omega, \tau)=\iint W_{E}\left(t^{\prime}, \omega^{\prime}\right) W_{R}\left(t^{\prime}-\tau, \omega-\omega^{\prime}\right) d t^{\prime} d \omega^{\prime} .
$$

The data can therefore be viewed in the chronocyclic space as a measurement of the overlap of the Wigner function of the pulse with the Wigner function of the gate (whose position in the space is related to the variables $\omega$ and $\tau$, which must vary over the entire region of phase space occupied by the pulse), and the latter therefore appears as the apparatus window function in spectrographic measurements. As a Wigner function has a lower bound of support in the time-frequency space (i.e., it always occupies a region of phase space greater than $\pi$ ), the spectrogram is always a "blurred" version of the Wigner function of the pulse. In principle the test pulse can be completely characterized very simply by direct Fourier deconvolution if the gate (and therefore its Wigner function) is known [17, 21, 26, 27]. In practice such deconvolution is highly sensitive to noise, since it involves the division of the Fourier transform of the measured spectrogram with the ambiguity function of the gate.
The spectrogram or sonogram can be used to obtain the instantaneous frequency and group delay of the signal. For example, the average frequency of the pulse for a given relative delay $\tau$ between the pulse and the gate provides a measure of the chirp, and can be obtained from the spectrogram via [21]

$$
\begin{aligned}
\langle\omega\rangle_{\tau} & =\frac{\int S(\omega, \tau) \omega d \omega}{\int S(\omega, \tau) d \omega} \\
& =-\frac{\int|E(t)|^{2}|R(t-\tau)|^{2}\left\{\varphi_{E}^{\prime}(t)+\varphi_{R}^{\prime}(t-\tau)\right\} d t}{\int S(\omega, \tau) d \omega} .
\end{aligned}
$$

If the gate function is real (i.e., it does not have a phase) and is much shorter than any variation of the electric field of the pulse under test, then it can be replaced in the integral with a Dirac delta function, and the average frequency calculated from the spectrogram approaches $-\varphi_{E}^{\prime}(\tau)$, that is, the instantaneous frequency of the electric field at the delay $\tau$. Such gate can be implemented by cross-correlating the pulse under test with a much shorter optical pulse without temporal phase distortion, though this is often impractical. The difficulty with this approach is that the uncertainty in the local average frequency becomes very large, since the spectral content of the spectrogram is dominated by the broad spectrum of the gate. Such approach was initially developed for short optical pulses $[28,29]$, and similar approaches are still being used in optical telecommunications [30, 31].

A better way to reconstruct the pulse field from a spectrogram is to use phase retrieval. In fact, this is the only option if the gate is unknown. The spectrogram of (20) is the modulus square of the short-time Fourier transform of the pulse. The trick in phase retrieval is to estimate the phase of the transform. Once this is known, a Fourier transform directly leads to the recovery of both the pulse under test and the gating function. Phase retrieval is often ambiguous in one dimension, but is usually unique in two dimensions [32]. The excess data available in the spectrogram enables iterative reconstruction of $N$ complex numbers specifying the field from the $N^{2}$ data points, and this can also lead to the simultaneous reconstruction of the gate $[23,33]$. Furthermore, in the case of the nonlinear spectrogram, there is often a known functional relation between the pulse and the gate, since the gate is implemented as a nonlinear interaction with replicas of the pulse under test. Also, other information might be available, such as the spectrum of the pulse or the transfer function of the gate. These constraints enable the recovery of the field by means of several algorithms. A very robust inversion algorithm is based on the principal component general projections algorithm [34], which uses projections derived from the 


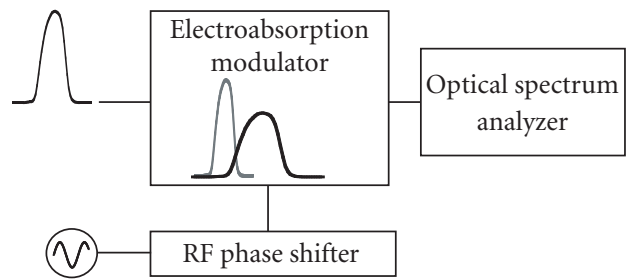

(a)

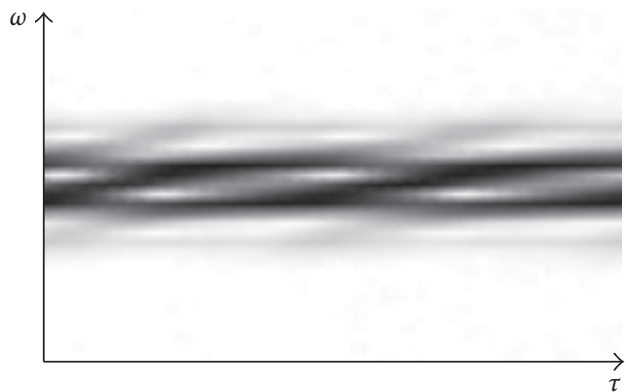

(b)

FiguRE 5: Example of the implementation of (a) spectrography and (b) spectrogram measured on a $40 \mathrm{GHz}$ alternate-chirp return-to-zero signal with an electroabsorption modulator driven at $10 \mathrm{GHz}$.

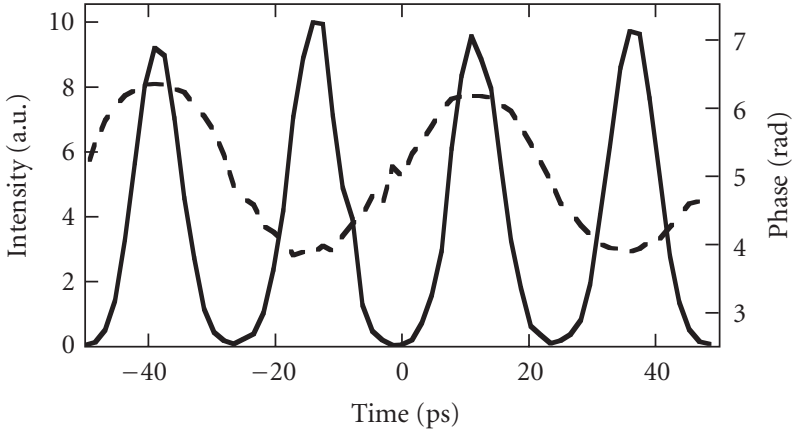

(a)

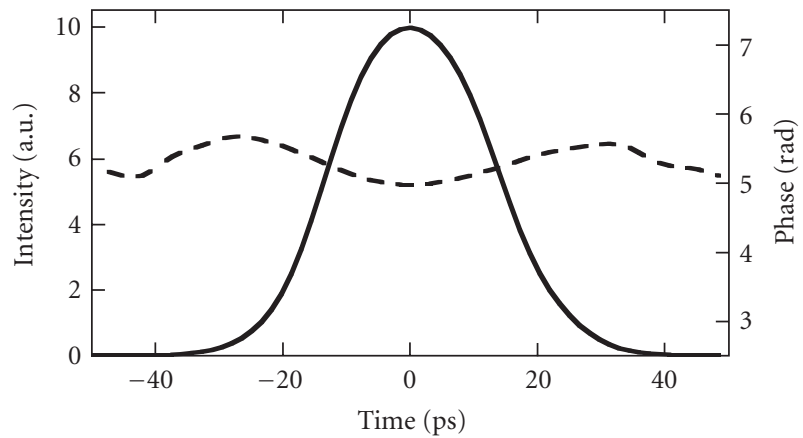

(b)

Figure 6: (a) Intensity and phase of the optical signal (resp., continuous and dashed line). (b) Transmission and phase of the gate (resp., continuous and dashed line) extracted from the previous spectrogram.

experimentally measured spectrogram and from the functional form of the spectrogram as the modulus square of the integral $\int E(t) R(t-\tau) \exp (i \omega t) d t$.

\subsection{Experimental spectrography for telecommunication applications}

Spectrograms and sonograms are popular tools for ultrashort optical pulse characterization. FROG generates a nonlinear spectrogram for the pulse by means of a nonlinear optical interaction of the pulse under test with one or several of its replicas [22]. This has the experimental advantage of using pump-probe geometries that are commonly used in ultrafast spectroscopy. Adaptations of FROG to pulse characterization in the telecommunication environment can be found, for example, in $[35,36,37,38]$.

For picosecond pulses, such as those used in telecommunications, a gate of smaller bandwidth than needed for femtosecond pulses, such as those typically found in ultrafast optics applications, suffices. It is possible to implement the gate using a temporal modulator, which has the important advantage of making the entire process linear. It is therefore extremely sensitive to small input pulse energies, yet insensitive to polarization and wavelength $[23,39]$.

The experimental implementation of spectrography with a temporal modulator is straightforward. The pulse under test is gated by a temporal modulator driven by a control signal synchronized to the pulse under test, as shown in Figure 5a. For example, an electroabsorption modulator can be driven by a sinusoidal voltage with well-defined phase relative to the test pulse. The relative delay $\tau$ between the signal under test and the gate is modified by changing the phase of the driving RF sine wave using a voltage-controlled phase shifter. The spectra after the modulator are recorded as a function of the optical frequency $\omega$ with a scanning monochromator, or with a Fabry-Perot etalon followed by a photodiode. An example of a measured spectrogram is displayed in Figure 5b. The characteristics of the pulse train and gate are extracted from the spectrogram, and examples of the retrieved signal and gate when characterizing an alternatechirp signal [40] are plotted in Figure 6.

\subsection{Interferometry}

Interferometry is a well-known approach to the characterization of optical fields in the spatial domain. It is a simple method for converting phase information into amplitude information that can then be read using square-law detectors. For temporal waveform measurement, interferometry, in contrast to spectrography, measures a single line of the two-time or two-frequency correlation function of the pulse under test. This is sufficient to characterize completely a 


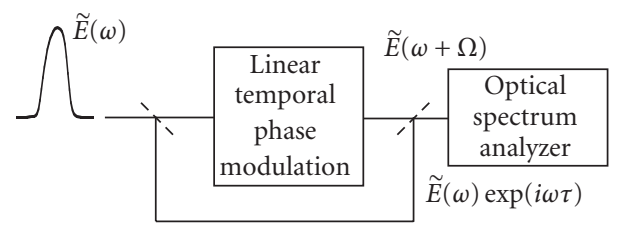

Figure 7: Conceptual approach for spectral shearing interferometry.

single pulse or a coherent pulse train, yielding $N$ samples of the field from $\alpha N$ data points $(\alpha>1)$. It therefore makes optimal use of the experimental data.

It is possible to construct the spectral equivalent of spatial shearing interferometry, in which the spatial phase profile of a beam is determined by interfering it with a laterally shifted (or sheared) replica. The resulting intensity interferogram can be measured with a square-law detector and the phase simply extracted. The spectral analogue, in which two spectrally sheared pulses are interfered, also allows direct reconstruction of the electric field in the spectral domain using the measured spectral phase and a pulse spectrum. Because a single slice of the correlation function is sufficient to characterize the electric field of a pulse that has a continuous spectral support, interferometry is viable for most applications. We will focus here on techniques that use the twotime frequency correlation function (or its sampled version) $\widetilde{E}(\omega) \widetilde{E}^{*}(\omega-\Omega)$, whose phase $\varphi(\omega)-\varphi(\omega-\Omega)$ can be concatenated or integrated to get the spectral phase of the initial pulse $[41,42]$. For a continuous spectral density, the spectral shear $\Omega$ is set by the sampling theorem, and it is typically a few percent of the total bandwidth of the pulse under test. Too large a shear would lead to undersampling of the pulse spectrum, while too small a shear could lead to increased sensitivity to noise, and thus reduced accuracy of the reconstruction. For a periodic source with high duty cycle, it suffices to measure the intensity and phase of the spectral modes, and the shear can usually be set to the value of the separation between the modes, that is, the repetition rate. The spectral intensity can be obtained either from a separate measurement using the spectrometer, or can be extracted from the correlation function directly.

A variety of interferometric techniques are known. The relative phase between adjacent spectral modes can, for example, be extracted in the time domain after spectral filtering $[43,44,45,46,47]$, or in the spectral domain after proper temporal modulation [48]. The latter class of techniques also includes spectral shearing interferometry, where the quantity $\widetilde{E}(\omega) \tilde{E}^{*}(\omega-\Omega)$ is obtained by measuring the interference of the pulse under test with its sheared replica with an optical spectrum analyzer (Figure 7). The frequency shear $\Omega$ can be implemented using a linear temporal phase modulation $\exp (i \Omega t)$. The spectral intensity of the two interfering pulses is $|\widetilde{E}(\omega)|^{2}+|\widetilde{E}(\omega-\Omega)|^{2}+\widetilde{E}(\omega) \widetilde{E}^{*}(\omega-\Omega)+\widetilde{E}^{*}(\omega) \widetilde{E}(\omega-\Omega)$. If a delay is introduced between the nonshifted and the shifted replica, this leads to spectral fringes with small spacing, by virtue of the phase $\varphi(\omega)-\varphi(\omega-\Omega)+\omega \tau$. In this case, the interferometric component can be directly extracted using Fourier processing of a single interferogram $[49,50]$.

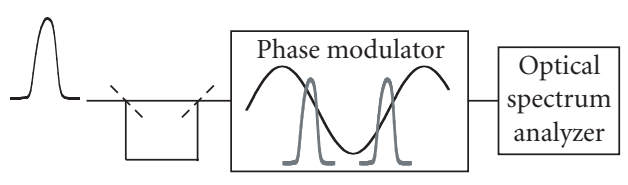

FIGURE 8: Experimental implementation of spectral shearing interferometry.

\subsection{Experimental interferometry for telecommunication applications}

There are numerous implementations of interferometry for the self-referencing characterization of optical pulses. For spectral shearing interferometry, a spectral shear of arbitrary value can be induced by mixing the test pulse with, for example, a highly chirped pulse in a nonlinear medium. This is known as spectral phase interferometry for direct electric field reconstruction [49]. A spectral shear can also be induced, as explained above, by linear temporal phase modulation of the pulse under test. Such modulation can be obtained using an electro-optic phase modulator driven by an appropriate voltage $[50,51]$. The generation of a strictly linear voltage at a high frequency is in practice difficult, and it is easier to use a sinusoidal voltage synchronized so that the pulse is at the zero-crossing of the modulation, therefore experiencing linear temporal phase modulation. One possible implementation is described in Figure 8.

The pulse under test is split into two replicas. These two replicas, separated by a delay $\tau$ are sent to a phase modulator driven by a sinusoidal voltage with period $2 \tau$. The synchronization is performed so that the two pulses stand at different zero-crossings of the modulation. Therefore, the pulses are sheared in opposite directions along the frequency axis. If $\Omega$ is the shear imposed on one of the pulses, the extracted spectral phase difference is $\varphi(\omega+\Omega)-\varphi(\omega-\Omega)+\omega \tau$. The carrier term $\omega \tau$ can be removed either by turning the modulation off [50] or by measuring a second phase difference when the relative pulse under test and the sine wave driving the modulator has been modified by $\tau$ [51]. In this case the extracted spectral phase is $\varphi(\omega-\Omega)-\varphi(\omega+\Omega)+\omega \tau$; so that the difference between the two extracted phases is $2 \varphi(\omega+\Omega)-2 \varphi(\omega-\Omega)$. Figure 9 displays an experimentally measured interferogram. The rapidly varying fringes due to the delay between the two interfering pulses are evident. An example of the intensity and phase measured using spectral shearing interferometry is also shown.

\subsection{Tomography}

As with spectrographic methods, the so-called tomographic techniques require in-series time-stationary and time-nonstationary filters so that the entire phase space can be explored. However, unlike spectrographic techniques, the first filter in a tomographic apparatus is a phase-only filter (either a quadratic temporal phase modulator or a quadratic spectral phase modulator). The inclusion of a quadratic phase-only filter results in a distinctly different interpretation of the measurement, leading to a fundamentally different 

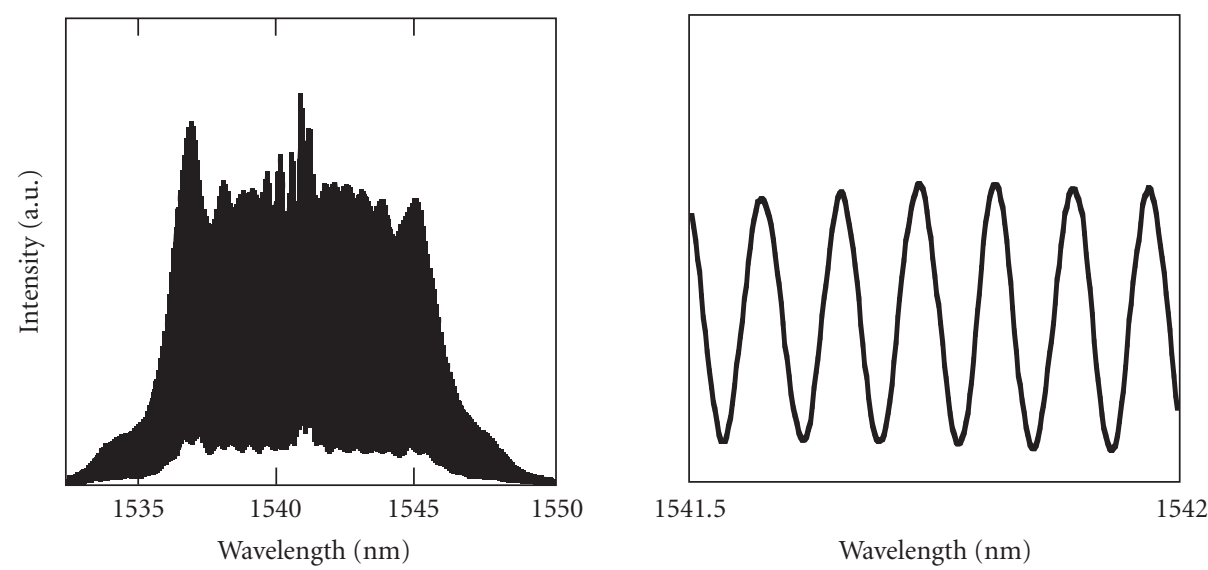

(a)

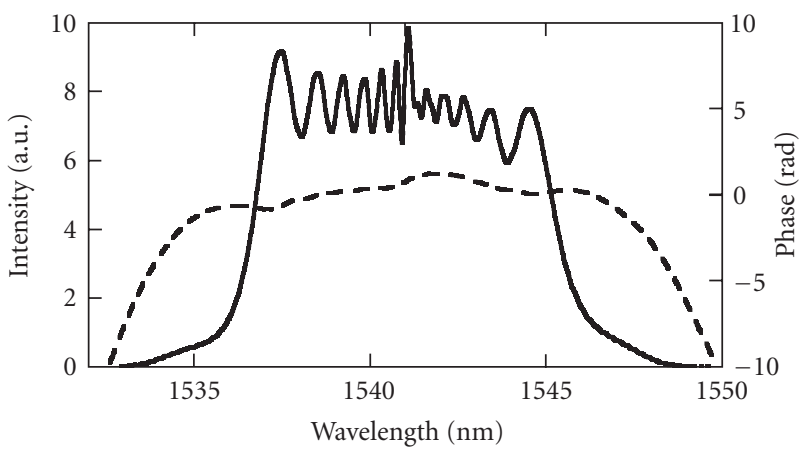

(b)

Figure 9: (a) Experimental interferogram and close-up on the fringes. (b) Reconstructed spectral intensity and phase (resp., continuous and dashed line).

inversion algorithm. To see this, notice that a phase-only filter does not provide any information on the frequency or the arrival time of a pulse ensemble and hence, does not constitute a measurement of either the spectral or temporal extent of the pulse. So, a tomographic apparatus does not make a simultaneous measurement of the conjugate variables time and frequency. Rather, the quadratic phase modulation acts to rotate the phase space. The square-law detector in combination with the amplitude-only filter records the resulting intensity distribution, that is, the projection of the rotated Wigner function. A sufficiently large number of phase-space rotations between $-\pi / 2$ and $\pi / 2$ allows, in principle, reconstruction of the Wigner function via the inverse Radon transform [52]. Numerical versions of this inversion algorithm were developed for applications of tomography in areas such as medical imaging, where one aims at reconstructing an object from a set of its projections (typically, 2D projections of a $3 \mathrm{D}$ object, or $1 \mathrm{D}$ projections of a $2 \mathrm{D}$ object) [53]. A typical implementation of chronocyclic tomography would use a combination of quadratic temporal and spectral phase modulations to rotate the phase space, and optical spectrum measurements to project the Wigner function. The Wigner function of the signal under test can be reconstructed from the measured projections, regardless of the degree of coherence of the pulse ensemble. This capability is unique to tomography among the techniques presented in this paper. This capability has not been realized experimentally, however, because of the relative difficulty of implementing variable temporal and spectral phase modulations.

The time-to-frequency converter $[54,55]$ operates by rotating the Wigner function by $\pi / 2$, so that a measurement of the frequency marginal after rotation leads to the temporal marginal, that is, one obtains the temporal intensity of the signal under test via an optical spectrum measurement. However, no phase information is obtained, and this approach requires a large rotation of the Wigner function, which is difficult to obtain.

The assumption that the pulse train is coherent, as is done in other methods, reduces the requirement on the number of projections needed to reconstruct the pulse field. This leads to the concept of simplified chronocyclic tomography. This technique does not require a large rotation of the phasespace density, and reconstructs the amplitude and phase of the signal from only two projections of the Wigner function. It is based on a particular relation between the frequency marginal of the rotated Wigner function and the electric field 


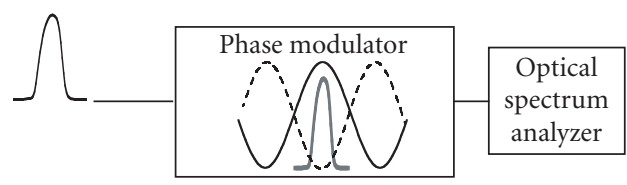

Figure 10: Experimental implementation of simplified chronocyclic tomography.

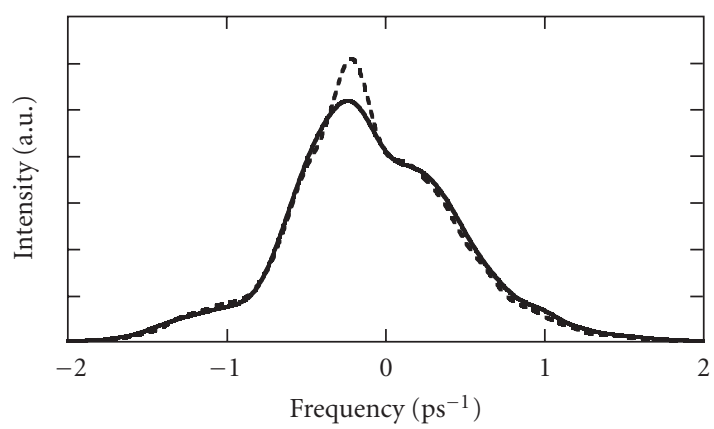

(a)

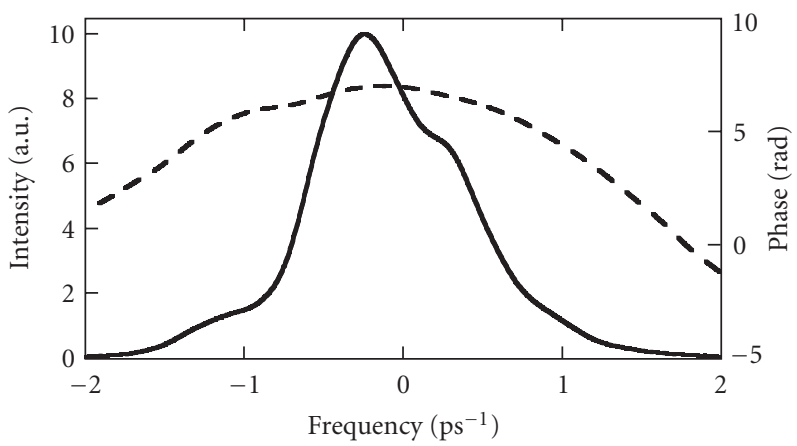

(b)

FIgURE 11: (a) Two spectra measured for small positive and negative quadratic temporal phase modulation (continuous and dashed line). (b) Reconstructed spectral intensity and phase (continuous and dashed line).

$[56,57]$. The fractional power spectrum of the pulse is obtained from the rotated Wigner function:

$$
I_{\alpha}(\omega)=\int W[t \cos (\alpha)+\omega \sin (\alpha), \omega \cos (\alpha)-t \sin (\alpha)] d t
$$

The derivative of this function with respect to the angle of rotation $\alpha$ at $\alpha=0$ leads to

$$
\frac{\partial I_{\alpha}}{\partial \alpha}=\int\left\lfloor\omega \frac{\partial W}{\partial t}-t \frac{\partial W}{\partial \omega}\right\rfloor d t=-\frac{\partial}{\partial \omega} \int t W d t
$$

and therefore to

$$
\frac{\partial I_{\alpha}}{\partial \alpha}=-\frac{\partial}{\partial \omega}\left\lfloor I \frac{\partial \varphi}{\partial \omega}\right\rfloor
$$

A rotation of the phase-space of the pulse requires a combination of a quadratic temporal and spectral phase modulations. However, the relation in (26) also holds for a shear of the phase-space, in which $\omega$ is transformed into $\omega+\psi t$, and the temporal coordinate is unchanged. This can be accomplished by means of a parabolic temporal phase modulation $(1 / 2) \psi t^{2}$ alone. In this case, one finds

$$
\frac{\partial I_{0}}{\partial \psi}=\frac{\partial}{\partial \psi} \int W(\omega+\psi t, t) d t=\frac{\partial}{\partial \omega}\left\lfloor I \frac{\partial \varphi}{\partial \omega}\right\rfloor
$$

This is the form most amenable to experiment, since the bandwidth required to generate a small shear using a phase modulator is modest.

\subsection{Experimental chronocyclic tomography}

Various implementations of the time-to-frequency converter have been performed using either a phase modulator or nonlinear optics. A phase modulator driven by a sinusoidal voltage can provide quadratic temporal phase modulation to a pulse synchronized with one of its extrema. A nonlinear interaction can also provide such modulation.

Simplified chronocyclic tomography has been implemented using a temporal phase modulator [57]. The pulse under test was synchronized with a maximum of the phase modulation, and the optical spectrum after modulation measured, as displayed in Figure 10. The pulse under test was then synchronized with a minimum of the phase modulation, and the corresponding optical spectrum measured. The derivative $\partial I_{0} / \partial \psi$ and the spectrum $I(\omega)$ are obtained, respectively, by taking the difference and the sum of the two measured spectra. Equation (26) is then used to reconstruct the phase $\varphi(\omega)$. Figure 11 displays an experimental pair of measured spectra and the reconstructed intensity and phase for a short optical pulse. The technique can also be improved using synchronous detection of the derivative $\partial I_{0} / \partial \psi$, which enables an increased signal-to-noise ratio [58].

\section{CONCLUSION}

Correlation functions and time-frequency distributions are important concepts for both representation and characterization of ultrashort optical pulses. Aside from their utility in analyzing the properties of the electric field of the pulse and for reconstructing the field from experimental data, they are necessary for representing the coherence properties of a train of optical pulses. The concepts of spectrography, tomography, and interferometry serve as useful major classifications 
for pulse-shape characterization, and each may be implemented using appropriate linear optical elements. For pulses typical in telecommunications applications, the use of linear temporal modulators as time-nonstationary filters in these classes of measurement has been detailed and experimental implementations for characterization in the telecommunication environment have been presented. As research in both signal analysis and ultrafast optics is in continual development, it can be expected that further exciting discoveries will be made in the near future.

\section{ACKNOWLEDGMENTS}

C. Dorrer would like to acknowledge his fruitful collaboration with Inuk Kang, from Bell Laboratories, Lucent Technologies, on the experiments described in $[23,39,50,51,57$, 58]. I. A. Walmsley acknowledges fruitful interactions with C. Iaconis and M. G. Raymer on methods of wave-field reconstruction. The work of I. A. Walmsley was supported by the US National Science Foundation and the UK Engineering and Physical Sciences Research Council.

\section{REFERENCES}

[1] I. Kaminow and T. Li, Optical Fiber Telecommunications IV, Academic Press, Boulder, Colo, USA, 2002.

[2] I. A. Walmsley, L. Waxer, and C. Dorrer, "The role of dispersion in ultrafast optics," Review of Scientific Instruments, vol. 72, no. 1, pp. 1-29, 2001.

[3] L. Lepetit, G. Cheriaux, and M. Joffre, "Linear techniques of phase measurement by femtosecond spectral interferometry for applications in spectroscopy," Journal of the Optical Society of America B, vol. 12, no. 12, pp. 2467-2474, 1995.

[4] D. N. Fittinghoff, J. L. Bowie, J. N. Sweetser, et al., "Measurement of the intensity and phase of ultraweak, ultrashort laser pulses," Optics Letters, vol. 21, no. 12, pp. 884-886, 1996.

[5] F. K. Fatemi, T. F. Carruthers, and J. W. Lou, "Characterisation of telecommunications pulse trains by fourier-transform and dual-quadrature spectral interferometry," Electronics Letters, vol. 39, no. 12, pp. 921-922, 2003.

[6] P. A. Andrekson, "Ultrahigh bandwidth optical sampling oscilloscopes," in Proc. Optical Fiber Communication Conference (OFC '04), Los Angeles, Calif, USA, February 2004, Tuo1.

[7] C. Dorrer, D. C. Kilper, H. R. Stuart, G. Raybon, and M. G. Raymer, "Linear optical sampling," IEEE Photon. Technol. Lett, vol. 15, no. 12, pp. 1746-1748, 2003.

[8] C. Dorrer, J. Leuthold, and C. R. Doerr, "Direct measurement of constellation diagrams of optical sources," in Proc. Optical Fiber Communication Conference (OFC '04), Los Angeles, Calif, USA, February 2004, PDP33.

[9] I. A. Walmsley and V. Wong, "Characterization of the electric field of ultrashort optical pulses," Journal of the Optical Society of America B, vol. 13, no. 11, pp. 2453-2463, 1996.

[10] C. Iaconis, V. Wong, and I. A. Walmsley, "Direct interferometric techniques for characterizing ultrashort optical pulses," IEEE J. Select. Topics Quantum Electron., vol. 4, no. 2, pp. 285294, 1998.

[11] J. W. Goodman, Introduction to Fourier Optics, McGraw-Hill, New York, NY, USA, 1996.

[12] S. A. Ponomarenko, G. P. Agrawal, and E. Wolf, "Energy spectrum of a nonstationary ensemble of pulses," Optics Letters, vol. 29, no. 4, pp. 394-396, 2004.

[13] M. Born and E. Wolf, Principles of Optics, Cambridge University Press, Cambridge, UK, 1998.
[14] K. H. Brenner and J. Ojeda-Castaneda, "Ambiguity function and Wigner distribution function applied to partially coherent imagery," Optica Acta, vol. 31, no. 2, pp. 213-223, 1984.

[15] T. A. C. M. Claasen and W. F. G. Mecklenbräuker, "The Wigner distribution-A tool for time-frequency signal analysis-Part III: relations with other time-frequency signal transformations," Philips Journal of Research, vol. 35, no. 6, pp. 372-389, 1980.

[16] K.-H. Brenner and K. Wodkiewicz, "The time-dependent physical spectrum of light and the wigner distribution function," Optics Communications, vol. 43, no. 2, pp. 103-106, 1982.

[17] J. Paye, "The chronocyclic representation of ultrashort light pulses," IEEE J. Quantum Electron., vol. 28, no. 10, pp. 22622273, 1992.

[18] C. A. Hirlimann and J.-F. Morhange, "Wavelet analysis of short light pulses," Applied Optics, vol. 31, no. 17, pp. 32633266, 1992.

[19] R. Gase, "Time-dependent spectrum of linear optical systems," Journal of the Optical Society of America A, vol. 8, no. 6, pp. 850-859, 1991.

[20] C. E. Cook and M. Bernfeld, Radar Signals: an Introduction to Theory and Application, Artech House, Norwood, Mass, USA, 1993, incorporated.

[21] L. Cohen, Time-Frequency Analysis, Prentice Hall PTR, Englewood Cliffs, NJ, USA, 1995.

[22] R. Trebino, Ed., Frequency-Resolved Optical Gating: the Measurement of Ultrashort Optical Pulses, Kluwer Academic, Boston, Mass, USA, 2002.

[23] C. Dorrer and I. Kang, "Simultaneous temporal characterization of telecommunication optical pulses and modulators by use of spectrograms," Optics Letters, vol. 27, no. 15, pp. 13151317, 2002.

[24] V. Wong and I. A. Walmsley, "Ultrashort-pulse characterization from dynamic spectrograms by iterative phase retrieval," Journal of the Optical Society of America B, vol. 14, no. 4, pp. 944-949, 1997.

[25] D. T. Reid, "Algorithm for complete and rapid retrieval of ultrashort pulse amplitude and phase from a sonogram," IEEE J. Quantum Electron., vol. 35, no. 11, pp. 1584-1589, 1999.

[26] V. Wong and I. A. Walmsley, "Phase retrieval in time-resolved spectral phase measurement", in Generation, Amplification, and Measurement of Ultrashort Laser Pulses II, vol. 2377 of Proceedings of SPIE, pp. 178-186, San Jose, Calif, USA, April 1995.

[27] K. Kikuchi, "Theory of sonogram characterization of optical pulses," IEEE J. Quantum Electron., vol. 37, no. 4, pp. 533-537, 2001.

[28] E. B. Treacy, "Measurement and interpretation of dynamic spectrograms of picosecond light pulses," Journal of Applied Physics, vol. 42, no. 10, pp. 3848-3858, 1971.

[29] J. L. A. Chilla and O. E. Martinez, "Analysis of a method of phase measurement of ultrashort pulses in the frequency domain," IEEE J. Quantum Electron., vol. 27, no. 5, pp. 12281235, 1991.

[30] K. Mori, T. Morioka, and M. Saruwatari, "Group velocity dispersion measurement using supercontinuum picosecond pulses generated in an optical fibre," Electronics Letters, vol. 29, no. 11, pp. 987-989, 1993.

[31] Y. Ozeki, Y. Takushima, and K. Kikuchi, "High dynamic range characterization of waveform and frequency-chirp of picosecond pulses based on the precise measurement of group delay in optical frequency domain," in Proc. Optical Fiber Communication Conference (OFC '03), vol. 2, pp. 725-727, Atlanta, Ga, USA, March 2003, FL3. 
[32] H. Stark, Ed., Image Recovery: Theory and Application, Academic Press, New York, NY, USA, 1987.

[33] D. J. Kane, G. Rodriguez, A. J. Taylor, and T. S. Clement, "Simultaneous measurement of two ultrashort laser pulses from a single spectrogram in a single shot," Journal of the Optical Society of America B, vol. 14, no. 4, pp. 935-943, 1997.

[34] D. J. Kane, "Real-time measurement of ultrashort laser pulses using principal component generalized projections," IEEE J. Select. Topics Quantum Electron., vol. 4, no. 2, pp. 278-284, 1998.

[35] M. D. Thomson, J. M. Dudley, L. P. Barry, and J. D. Harvey, "Completepulse characterization at $1.5 \mu \mathrm{m}$ by cross-phase modulation in optical fibers," Optics Letters, vol. 23, no. 20, pp. 1582-1584, 1998.

[36] K. Ogawa and M. D. Pelusi, "High-sensitivity pulse spectrogram measurement using two-photon absorption in a semiconductor at 1.5- $\mu \mathrm{m}$ wavelength," Optics Express, vol. 7, no. 3, pp. 135-140, 2000.

[37] P.-A. Lacourt, J. M. Dudley, J.-M. Merolla, H. Porte, J.P. Goedgebuer, and W. T. Rhodes, "Milliwatt-peak-power pulse characterization at $1.55 \mu \mathrm{m}$ by wavelength-conversion frequency-resolved optical gating," Optics Letters, vol. 27, no. 10, pp. 863-865, 2002.

[38] L. P. Barry, S. Del Burgo, B. Thomsen, R. T. Watts, D. A. Reid, and J. Harvey, "Optimization of optical data transmitters for $40 \mathrm{~Gb} / \mathrm{s}$ lightwave systems using frequency resolved optical gating," IEEE Photon. Technol. Lett., vol. 14, no. 7, pp. 971973, 2002.

[39] C. Dorrer and I. Kang, "Real-time implementation of linear spectrograms for the characterization of high bit-rate optical pulse trains," IEEE Photon. Technol. Lett., vol. 16, no. 3, pp. 858-860, 2004.

[40] P. J. Winzer, C. Dorrer, R.-J. Essiambre, and I. Kang, "Chirped return-to-zero modulation by imbalanced pulse carver driving signals," IEEE Photon. Technol. Lett., vol. 16, no. 5, pp. 1379-1381, 2004.

[41] V. A. Zubov and T. I. Kuznetsova, "Solution of the phase problem for time-dependent optical signals by an interference system," Soviet Journal of Quantum Electronics, vol. 21, pp. 1285 1286, 1991.

[42] V. Wong and I. A. Walmsley, "Analysis of ultrashort pulseshape measurement using linear interferometers," Optics Letters, vol. 19, no. 4, pp. 287-289, 1994.

[43] K. C. Chu, J. P. Heritage, R. S. Grant, et al., "Direct measurement of the spectral phase of femtosecond pulses," Optics Letters, vol. 20, no. 8, pp. 904-906, 1995.

[44] P. Kockaert, M. Peeters, S. Coen, Ph. Emplit, M. Haelterman, and O. Deparis, "Simple amplitude and phase measuring technique for ultrahigh-repetition-rate lasers," IEEE Photon. Technol. Lett, vol. 12, no. 2, pp. 187-189, 2000.

[45] M. Kwakernaak, R. Schreieck, A. Neiger, H. Jackel, E. Gini, and W. Vogt, "Spectral phase measurement of mode-locked diode laser pulses by beating sidebands generated by electrooptical mixing," IEEE Photon. Technol. Lett., vol. 12, no. 12, pp. 1677-1679, 2000.

[46] P. Kockaert, J. Azana, L. R. Chen, and S. LaRochelle, "Full characterization of uniform ultrahigh-speed trains of optical pulses using fiber Bragg gratings and linear detectors," IEEE Photon. Technol. Lett., vol. 16, no. 6, pp. 1540-1542, 2004.

[47] P. Kockaert, M. Haelterman, Ph. Emplit, and C. Froehly, "Complete characterization of (ultra)short optical pulses using fast linear detectors," IEEE J. Select. Topics Quantum Electron., vol. 10, no. 1, pp. 206-212, 2004.
[48] J. Debeau, B. Kowalski, and R. Boittin, "Simple method for the complete characterization of an optical pulse," Optics Letters, vol. 23, no. 22, pp. 1784-1786, 1998.

[49] C. Iaconis and I. A. Walmsley, "Spectral phase interferometry for direct electric-field reconstruction of ultrashort optical pulses," Optics Letters, vol. 23, no. 10, pp. 792-794, 1998.

[50] C. Dorrer and I. Kang, "Highly sensitive direct characterization of femtosecond pulses by electro-optic spectral shearing interferometry," Optics Letters, vol. 28, no. 6, pp. 477-479, 2003.

[51] I. Kang, C. Dorrer, and F. Quochi, "Implementation of electro-optic spectral shearing interferometry for ultrashort pulse characterization," Optics Letters, vol. 28, no. 22, pp. 2264-2266, 2003.

[52] M. Beck, M. G. Raymer, I. A. Walmsley, and V. Wong, "Chronocyclic tomography for measuring the amplitude and phase structure of optical pulses," Optics Letters, vol. 18, no. 23, pp. 2041-2043, 1993.

[53] A. C. Kak and M. Slaney, Principles of Computerized Tomographic Imaging, IEEE Press, New York, NY, USA, 1988.

[54] M. T. Kauffman, W. C. Banyai, A. A. Godil, and D. M. Bloom, "Time-to-frequency converter for measuring picosecond optical pulses," Applied Physics Letters, vol. 64, no. 3, pp. 270272, 1994.

[55] L. Kh. Mouradian, F. Louradour, V. Messager, A. Barthélémy, and C. Froehly, "Spectro-temporal imaging of femtosecond events," IEEE J. Quantum Electron., vol. 36, no. 7, pp. 795$801,2000$.

[56] T. Alieva, M. J. Bastiaans, and L. Stankovic, "Signal reconstruction from two close fractional Fourier power spectra," IEEE Trans. Signal Processing, vol. 51, no. 1, pp. 112-123, 2003.

[57] C. Dorrer and I. Kang, "Complete temporal characterization of short optical pulses by simplified chronocyclic tomography," Optics Letters, vol. 28, no. 16, pp. 1481-1483, 2003.

[58] I. Kang and C. Dorrer, "Highly sensitive differential tomographic technique for real-time ultrashort pulse characterization," in Proc. Conference on Lasers and Electro-Optics (CLEO '04), vol. 1, San Francisco, Calif, USA, May 2004, CTuZ6.

Christophe Dorrer is a Member of Technical Staff at Bell Laboratories, Lucent Technologies. He has a Ph.D. degree in optics from the École Polytechnique, France. He works in various domains of ultrafast optics related to optical telecommunications, such as optical pulse characterization, characterization of high-bit-rate dataencoded sources, characterization of devices and fibers, pulse shaping, and monitoring of optical networks.

Ian A. Walmsley is the Hook Professor of expermental physics at the University of Oxford, and the Head of the Department of Atomic and Laser Physics, Clarendon Laboratory. He has a Ph.D. degree in optics from the University of Rochester. His research concerns quantum processes on ultrafast time scales, and he has developed methods for the generation and measurement of nonclassical states of light and mat-

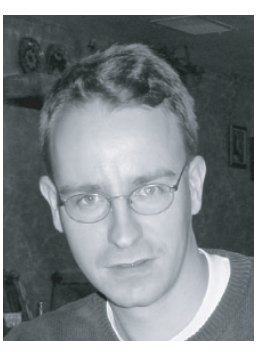
ter, as well as contributed to ultrafast technology through the invention of methods for ultrashort pulse characterization. 